\title{
The validity of the patient health Questionnaire-9 to screen for depression in patients with type-2 diabetes mellitus in non-communicable diseases clinics in Malawi
}

Michael Udedi ${ }^{1,2,3^{*}}$ (D), Adamson S. Muula ${ }^{3,4}$, Robert C. Stewart ${ }^{1}$ and Brian W. Pence ${ }^{5}$

\begin{abstract}
Background: Depression is a global problem, affecting populations worldwide, but is too often under-diagnosed. The identification of depression among patients with diabetes is important because depression is prevalent in this group and can complicate diabetes management.

Objectives: The aim of the study was to determine the sensitivity and specificity of the PHQ-9 in the detection of depression among patients with type-2 diabetes mellitus attending non-communicable diseases (NCD) clinics in Malawi.

Methods: We conducted a validation study of the Patient Health Questionnaire (PHQ-9) among 323 patients with type-2 diabetes mellitus who attended two NCD clinics in one of the 28 districts of Malawi. The participants were screened consecutively using the nine-item PHQ-9 in Chichewa by a research assistant and completed a diagnostic interview using the Structured Clinical Interview for DSM-IV (SCID) for depression with a mental health clinician. We evaluated both content validity based on expert judgement and criterion validity of the Patient Health Questionnaire (PHQ-9) based on performance against the SCID. The PHQ-9 cutpoint that maximized sensitivity plus specificity was selected to report test characteristics.
\end{abstract}

Results: Using the SCID for depression, the prevalence of minor or major depression was 41\% (133/323). The internal consistency estimate for the PHQ-9 was 0.83, with an area under the receiver operator curve (AUC) of 0.93 ( $95 \% \mathrm{Cl}$, [0. 91-0.96]). Using the optimal cut-point of $\geq 9$, the PHQ-9 had a sensitivity of $64 \%$ and a specificity of $94 \%$ in detecting both minor and major depression, with likelihood ratio-positive $=10.1$ and likelihood ratio negative $=0.4$ as well as overall correct classification (OCC) rate of 81\%.

Conclusions: This is the first validation study of the PHQ-9 in NCD clinics in Malawi. Depression was highly prevalent in this sample. The PHQ-9 demonstrated reasonable accuracy in identifying cases of depression and is a useful screening tool in this setting. Health care workers in NCD clinics can use the PHQ-9 to identify depression among their patients with those having a positive screen followed up by additional diagnostic assessment to confirm diagnosis.

Trial registration: PACTR201807135104799. Retrospectively registered on 12 July 2018.

Keywords: PHQ-9, Validation, Non communicable diseases, Depression, Diabetes mellitus, Malawi

\footnotetext{
* Correspondence: mphatsoudedi@yahoo.co.uk

'Department of Mental Health, University of Malawi, College of Medicine, P/

Bag 360, Chichiri, Blantyre 3, Malawi

${ }^{2}$ Department of Clinical Services, Ministry of Health, P. O. Box 30377, Capital

City, Lilongwe 3, Malawi

Full list of author information is available at the end of the article
}

(c) The Author(s). 2019 Open Access This article is distributed under the terms of the Creative Commons Attribution 4.0 International License (http://creativecommons.org/licenses/by/4.0/), which permits unrestricted use, distribution, and reproduction in any medium, provided you give appropriate credit to the original author(s) and the source, provide a link to the Creative Commons license, and indicate if changes were made. The Creative Commons Public Domain Dedication waiver (http://creativecommons.org/publicdomain/zero/1.0/) applies to the data made available in this article, unless otherwise stated. 


\section{Background}

Depression contributes significantly to the global burden of disease. Depression is often under-diagnosed in low and middle-income countries (LMICs) [1, 2]. The poor detection of depression is associated with disability and leads to increased use of health services for physical health complaints in both high-income countries and LMICs [3]. If healthcare workers are able to diagnose depression, they can reduce morbidity and improve patient wellbeing by providing cost-effective treatments $[4,5]$. In order to detect and diagnose depression, we need to have effective tools. Because depression varies based on cultural context, screening tools must be adapted and validated for particular populations.

There are a number of tools used to screen for depression. The Patient Health Questionnaire (PHQ-9) is widely used for screening and monitoring treatment of depression [6]. The PHQ 9 is a nine-item scale assessing symptoms experienced in the preceding two weeks. The reliability and validity of the PHQ-9 are sound, and internal validity of the PHQ-9 is high. The PHQ-9 questions are easily understood, and the PHQ-9 requires minimal time to administer and score [7]. The PHQ-9 has been validated and translated in some African countries including Nigeria [8], Ghana [9], Kenya [10], Cameroon, [11], Ethiopia [12], South Africa [13], and Uganda [1]. However, the PHQ-9 has not been validated for use in Malawi, and there are currently few tools for efficient and effective depression screening in a general healthcare setting in Malawi. The Self Reporting Questionnaire (SRQ) has been validated to screen for major depressive episodes in Malawi [14]. However the SRQ-20 is limited in response options ('yes' or 'no') [15], whereas the PHQ-9 has greater variety of options for describing symptom occurrence.

Accordingly, we conducted a validation study of the PHQ-9 for detection of depression in Malawi. We validated this tool in patients with type-2 diabetes mellitus attending two NCD clinics in Malawi. We chose the PHQ-9 for this study because it is effective in other settings and brief, which is compatible with Malawian health care setting workload. We conducted this study in an NCD clinic because depression is often comorbid with NCDs [16-18], and it is particularly important to have a tool that is validated for use in general healthcare settings, such as an NCD clinic. We included only patients with diabetes because the health care burden associated with the rapidly increasing diabetes population in Malawi, and the concern that depression can interfere with clinic appointment attendance and treatment adherence, makes this a timely and important focus [19-23]. The use of a valid screening tool for depression will help clinicians better diagnose patients and initiate treatment, which is in line with the strategy of integration of mental health as outlined in Malawi's National Mental Health Policy.

\section{Materials and methods}

\section{Setting and participants}

We conducted the study in Lilongwe district, a predominantly Chichewa speaking district. The study was conducted in two NCD clinics of Area 25 Health Centre under Lilongwe District Health Office and Kamuzu Central Hospital. The Area 25 Health Centre has been piloting a chronic care clinic for key NCDs such as hypertension, diabetes, asthma and epilepsy at the primary health care level since March 2014. Research assistants recruited consecutive patients attending the NCD clinic at the study sites between December 2017 and April 2018. Participants were eligible for the study if they were at least 18 years of age or older, attending the NCD clinic for diabetes care, and available for an interview. Participants were excluded if they required acute medical care or were unable to speak.

\section{Validation \\ Content validity}

The PHQ-9 is a depression module that incorporates the Diagnostic and Statistical Manual of Mental Disorders (DSM) criteria into a brief measure of depression [24]. The PHQ-9 is a concise tool for assessing depression. Two bilingual Malawians translated the English PHQ-9 independently into Chichewa; one mental health nurse and one linguistics and communication specialist. This was followed by evaluation of the translated tools by the principal investigator, two mental health professionals and two health promotion officers with extensive expertise in developing health communication tools in order to arrive at a consensus translation. Two additional independent bilingual Malawians back-translated the consensus Chichewa translation into English.

We pretested the Chichewa translated version of the PHQ 9 on a convenience small sample of 15 participants attending a general outpatient clinic in area 25. The PHQ-9 was interviewer-administered, and participants were probed about their perceived interpretation of the constructs. This pretesting helped to identify any challenges respondents might have with the translation. Any unclear Chichewa terms were modified to include terms that are more commonly used and understandable by the participants in order to produce a final PHQ-9 Chichewa translation. (Additional file 1).

\section{Criterion validity}

The Structured Clinical Interview for DSM-IV (SCID) [25] depression module was used as a gold standard to validate the PHQ 9. The SCID is a semi-structured interview designed for administration by a clinician or skilled researcher that determines formal diagnosis according to the Diagnostic and Statistical Manual of Mental Disorders. The SCID for depression was translated into Chichewa previously and has been used in Malawi after 
undergoing a process of validation which included translation, back translation and testing [14] . At the time of the study the most recent version of SCID was not available however major depression is defined in both the DSM-IV and DSM-V as the presence of either depressed mood or loss of interest or irritability with five or more depressive symptoms, lasting at least two weeks, with no history of a manic, hypomanic, or mixed episode [26, 27]. In contrast, minor depression is described in the DSM-IV as the presence of at least two, but less than five, depressive symptoms (one symptom must be either depressed mood or loss of interest) during the same 2-week period, with no history of a major depressive episode or dysthymia [27].

\section{Study procedure}

The sample size was calculated using Buderer's formula [28]. We used the following parameters: anticipated sensitivity $(S N)$ of the PHQ-9 was $80 \%$, the standard normal deviation corresponding to the specified size of the critical region $\left(\mathrm{z}^{2}{ }^{-\alpha / 2}\right)$ is 3.84 , alpha $(\alpha)$ which is size of the critical region is 0.05 , the absolute precision desired on either side of sensitivity L was set at 0.1 , and prevalence for depression was estimated as $20 \%$. The prevalence of depression of $20 \%$ was based on a recent study among patients attending a health care setting in Malawi [29]. We increased the sample size by $5 \%$ to account for potential participants' refusal and loss of data. Taking these assumptions into consideration, the required sample size was calculated as 323 .

Two research assistants conducted the recruitment and screening of participants. The research assistants had bachelor's degree and had 3 days of training in administration of the research tools and spoke both English and Chichewa. After assessing each patient for inclusion and exclusion criteria, the research assistants explained the study's purpose and procedures. Informed consent (signed or thumb printed) was obtained from patients. The research assistant collected demographic information from the participant and then administered the Chichewa version of the PHQ-9 in a private room.

After seeing their clinician for their regular appointment, participants completed the SCID with a separate SCID interviewer. The SCID interviewer was masked from the PHQ-9 score. The SCID interviewer was a mental health clinician and had regular supervision and record review for quality assurance to ensure consistence and accuracy of diagnoses. Finally, the patient's health passport was examined to determine whether or not the NCD clinician had made a diagnosis of depression and/or prescribed antidepressant medication during the clinical encounter.

\section{Data analyses}

We compared the PHQ-9 score against the "SCID" diagnosis of depression that was made by the 2-stage diagnostic process. We also calculated the sensitivity, specificity, positive predictive value (PPV), and negative predictive value (NPV) for various PHQ-9 cut-off scores. The PHQ-9 ability to discriminate between cases and noncases was then examined using receiver operating characteristic (ROC) analysis. The ROC curve analysis was used to choose cut-points for the PHQ-9 scale. Two different cut-points were used, and the diagnostic ability was assessed by a number of statistics at each of these points. The first cut-point was the point which maximised the combination of sensitivity and specificity, whilst the second cut-point was chosen to give a more give a higher sensitivity. The PHQ-9 cutpoint that maximized sensitivity + specificity was selected to report test characteristics. The ROC curves were obtained by plotting sensitivity against 1-specificity for each possible cut-off score. The area under the ROC curve (AUC) was used to indicate the performance of the PHQ-9. A value of 0.5 on the AUC indicates discrimination no better than chance, and a value of 1.0 represents perfect discrimination. The correctly classified rate and likelihood ratio were also considered. Internal consistency of the PHQ-9 was evaluated using Cronbach's alpha. Data analysis was conducted using SPSS version 20.0.

\section{Ethical approval}

We obtained ethical approval from the University of Malawi, College of Medicine Review and Ethics Committee (COMREC) (Reference-P.07/17/2218). Written informed consent was obtained from every participant and fingerprint impressions were taken from consenting illiterate participants. All interviews were conducted in private at the health facilities.

\section{Results \\ Sample characteristics}

In total, 323 patients who were eligible and approached completed both the PHQ-9 and SCID between December, 2017 and April, 2018 thus we had no refusals. Among the 323 patients, 127 (39.3\%) had diabetes only while 196 (60.7\%) had both diabetes and hypertension. The mean patient age was 54 years (range, $21-79$ years), with a standard deviation of 11.4 years; $75.5 \%$ of patients were female (Table 1).

\section{Prevalence of depression}

Of the 323 patients, a total of 133 had either a minor or major depression identified by SCID, resulting in an estimated prevalence of $41 \%$. Major depression was observed in $58(18 \%)$ of the patients. No patients $(0 \%)$ were diagnosed with depression or prescribed depression treatment by the NCD clinicians. 
Table 1 Socio-demographic characteristics of 323 validation study participants recruited from two non-communicable diseases clinics in Lilongwe, Malawi

\begin{tabular}{ll}
\hline Age & Mean (SD) \\
\hline Mean age in years & $53.8(11.4)$ \\
Gender & $\mathrm{n}(\%)$ \\
Female & $244(75.5)$ \\
Male & $79(24.5)$ \\
Education & \\
No education & $27(8.4)$ \\
Primary school & $153(47.4)$ \\
Secondary & $127(39.3)$ \\
Tertiary & $16(5.0)$ \\
Marital status & \\
Married & $249(77.0)$ \\
Never married & $8(2.5)$ \\
Widowed & $50(15.5)$ \\
Separated/divorced & $16(5.0)$ \\
Employment Status & \\
Unemployed & $179(55.4)$ \\
Student & $1(0.3)$ \\
Employed & $64(19.8)$ \\
Micro \& Small scale Enterprise & $79(24.5)$ \\
Religious affiliation & \\
Christian & $305(94.4)$ \\
Moslem & $17(5.3)$ \\
None & $1(0.3)$ \\
\hline & \\
\hline
\end{tabular}

\section{Performance of the patient health Questionnaire-9}

The calculated internal consistency of the PHQ-9 (Cronbach's alpha) was 0.83 . The area under the receiver operator curve was 0.93 (95\% CI, [0.91-0.96]) (Fig. 1), suggesting good discriminating power of the PHQ-9 between cases and non cases of depression.

The results suggested that a cut-point of 6 or higher on the PHQ-9 scale gave the best combination of sensitivity and specificity in detecting either minor or major depression. This cut-point gave a very high sensitivity of $93 \%$ and a lower specificity of $78 \%$. There was a high NPV of $94 \%$, but a lower PPV of $74 \%$. The Overall Correct Classification (OCC) rate was $84 \%$ at a cut point of 6 or higher and the likelihood ratio of a positive screen for depression was 4.2) and the likelihood ratio of a negative screen was 0.9 . Using a higher cut-off of $\geq 7$ increased the specificity to $86 \%$. However, this was at the expense of sensitivity, which dropped down to $80 \%$. There was a high NPV of $86 \%$, but a lower PPV of $79 \%$ with an OCC of $83 \%$ and likelihood ratio-positive of 5.6.

The best combination of sensitivity and specificity was found to be at a cut-point of 9 or higher. A cut-point of $\geq 9$ had a sensitivity of $64 \%$ and a specificity of $94 \%$ in detecting either minor or major depression. At the cut-point of $\geq 9$, the NPV was $79 \%$ while the PPV was $88 \%$ and the likelihood ratio-positive was 10.1 and the likelihood ratio-negative was 0.4 . The OCC rate of $81 \%$ was also good at a cut point of 9 and higher (Table 2).

A similar analysis was performed to examine the predictive ability of PHQ-9 for detecting major depression alone. The ROC curve analysis gave an AUC value of 0.91 (95\% CI, 0.88 to 0.94; Fig. 2).

The best combination of sensitivity and specificity was found to be at a cut-point of 9 or higher. This gave a relative high sensitivity and specificity of 85 and $82 \%$ respectively. There was also very high NPV with a value of

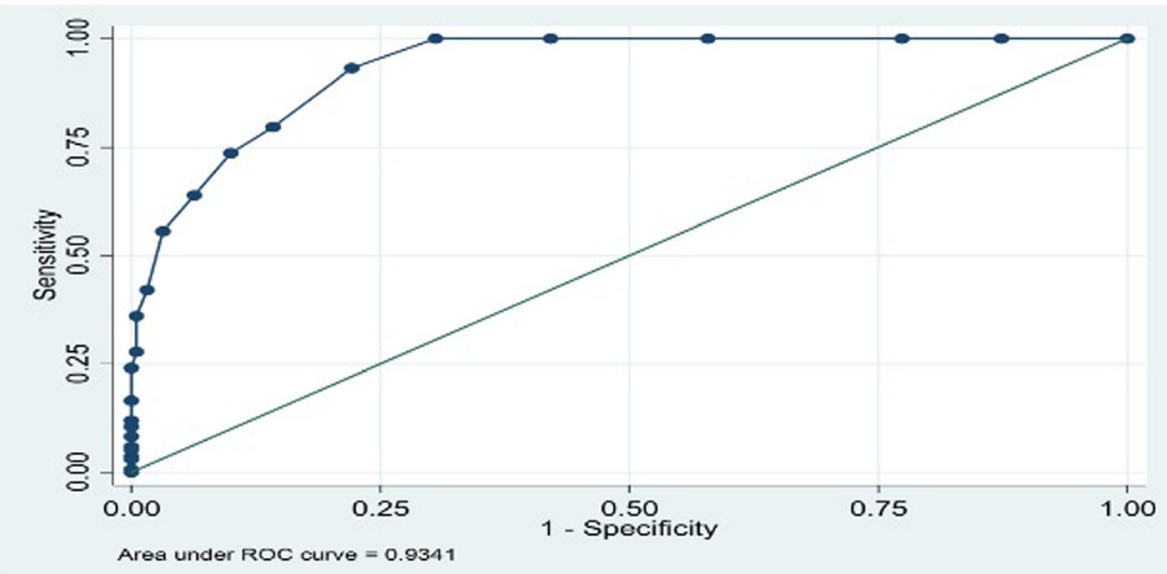

Fig. 1 The predictive ability of $\mathrm{PHQ}-9$ for detecting any depression 
Table 2 Operating characteristics of the Patient Health Questionnaire-9 at various cut-off scores for identifying either minor or major depression

\begin{tabular}{llllll}
\hline Cut point & Sensitivity & Specificity & Correctly Classified & LR+ & LR - \\
\hline$(>=4)$ & $100.00 \%$ & $57.89 \%$ & $75.23 \%$ & 2.3750 & 0.0000 \\
$(>=5)$ & $100.00 \%$ & $69.47 \%$ & $82.04 \%$ & 3.2759 & 0.0000 \\
$(>=6)$ & $93.23 \%$ & $77.89 \%$ & $84.21 \%$ & 4.2177 & 0.0869 \\
$(>=7)$ & $79.70 \%$ & $85.79 \%$ & $83.28 \%$ & 5.6085 & 0.2366 \\
$(>=8)$ & $73.68 \%$ & $90.00 \%$ & $83.28 \%$ & 7.3684 & 0.2924 \\
$(>=9)$ & $63.91 \%$ & $93.68 \%$ & $81.42 \%$ & 10.1190 & 0.3852 \\
$(>=10)$ & $55.64 \%$ & $96.84 \%$ & $79.88 \%$ & 17.6190 & 0.4581 \\
$(>=11)$ & $42.11 \%$ & $98.42 \%$ & $75.23 \%$ & 26.6668 & 0.5882 \\
$(>=12)$ & $36.09 \%$ & $99.47 \%$ & $73.37 \%$ & 68.5717 & 0.6425 \\
$(>=13)$ & $27.82 \%$ & $99.47 \%$ & $69.97 \%$ & 52.8573 & 0.7256 \\
\hline L $+=$ Likelihood Ratio Positive LR- = Likelihood Ratio Negative &
\end{tabular}

$96 \%$, but a lower PPV of $51 \%$ with an OCC of $82 \%$ and likelihood ratio-positive of 4.6. Lowering the cut-point on the PHQ-9 scale to 7 gave relatively similar overall performance in terms of the combination of sensitivity and specificity. However, using this cut-off increased sensitivity up to $95 \%$, but at the expense of specificity which dropped to $71 \%$. The OCC was $75 \%$ and likelihood ratio-positive was 3.2 (Table 3 ).

\section{Discussion}

Few screening tool to detect common mental disorders (CMDs) have been specifically developed in low and middle income countries [30] as such many researchers rely on tools from developed countries. It is important that instruments for screening patients have to be evaluated for their reliability and validity prior to their use in
Table 3 Operating characteristics of the Patient Health Questionnaire-9 at various cut-off scores for identifying major depression

\begin{tabular}{llllll}
\hline Cut point & Sensitivity & Specificity & Correctly Classified & LR+ & LR - \\
\hline$(>=4)$ & $100.00 \%$ & $41.51 \%$ & $52.01 \%$ & 1.7097 & 0.0000 \\
$(>=5)$ & $100.00 \%$ & $49.81 \%$ & $58.82 \%$ & 1.9925 & 0.0000 \\
$(>=6)$ & $100.00 \%$ & $59.25 \%$ & $66.56 \%$ & 2.4537 & 0.0000 \\
$(>=7)$ & $94.83 \%$ & $70.57 \%$ & $74.92 \%$ & 3.2217 & 0.0733 \\
$(>=8)$ & $89.66 \%$ & $75.47 \%$ & $78.02 \%$ & 3.6552 & 0.1371 \\
$(>=9)$ & $84.48 \%$ & $81.89 \%$ & $82.35 \%$ & 4.6642 & 0.1895 \\
$(>=10)$ & $72.41 \%$ & $85.66 \%$ & $83.28 \%$ & 5.0499 & 0.3220 \\
$(>=11)$ & $67.24 \%$ & $92.45 \%$ & $87.93 \%$ & 8.9095 & 0.3543 \\
$(>=12)$ & $55.17 \%$ & $93.58 \%$ & $86.69 \%$ & 8.6004 & 0.4790 \\
$(>=13)$ & $46.55 \%$ & $95.85 \%$ & $87.00 \%$ & 11.2147 & 0.5576 \\
\hline LR+ L Likelihood Ratio Positive LR- = Likelihood Ratio Negative &
\end{tabular}

a country to ensure that the instruments are measuring what they supposed to measure [31].

In this study when the PHQ-9 was used against the gold-standard diagnosis, it performed well showing reasonable accuracy in identifying cases of depression. The area under the ROC curve was found to be 0.93 , this is a high value, suggesting good diagnostic ability of the PHQ-9 score.

In this study, the PHQ-9 showed good predictive performance, comparable to that seen in validation studies in other parts of sub-Saharan Africa $[1,13]$. The internal consistency observed for the PHQ-9 was also similar to that found in other previous study elsewhere [32]. Maximum sensitivity with a specificity $\geq 75 \%$ has been considered as desirable for clinical use [32]. Relative to previous studies, our results suggest PHQ-9 has better sensitivity and acceptable specificity in the type 2 diabetic population.

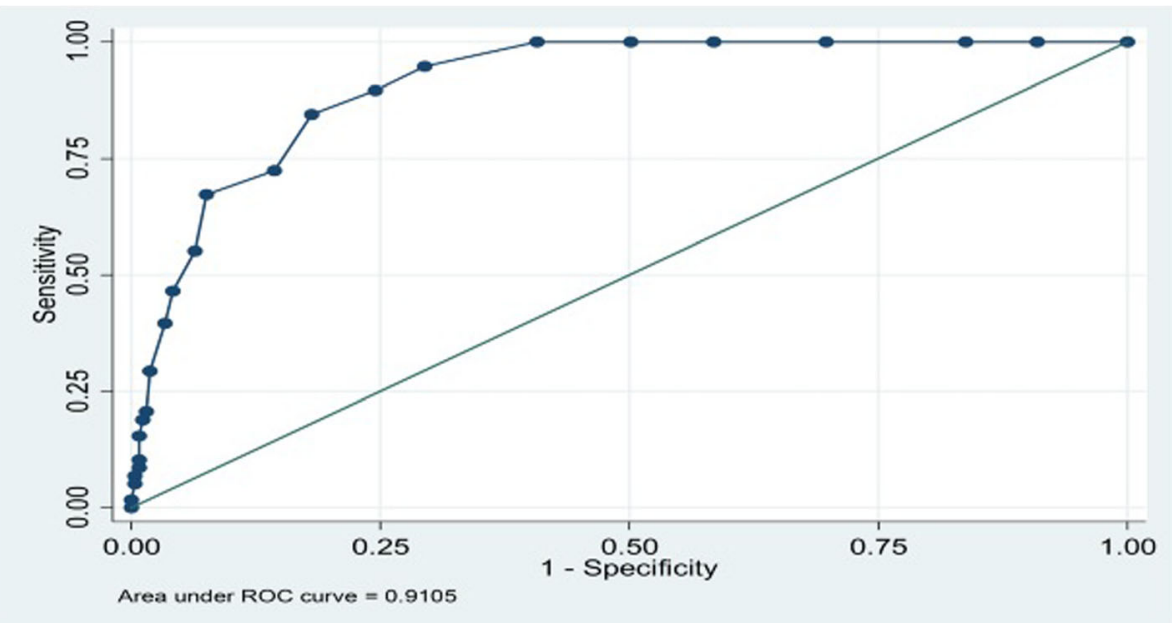

Fig. 2 The predictive ability of PHQ-9 for detecting Major Depression 
Depression is common and likely to impact diabetes mellitus care therefore routine screening is really important in diabetes mellitus care and we need these tools. In this validation study of the PHQ-9 in NCD clinic settings in Malawi, there was evidence of high prevalence of depression in patients with diabetes mellitus. The rate of depression in this study is comparable to rates of depression in other LMICs such as $46 \%$ in South Africa, 40\% in Iraq, 32\% in Egypt, 15 to 30\% in Nigeria, 14. 7 to $43 \%$ in Pakistan, 43 to $70 \%$ in Iran, 27 to $63 \%$ in Mexico [33] and 39.73\% in Ethiopia [34]. Depression often is missed by clinicians working in NCD clinics. Indeed, none of the cases of SCID-defined minor or major depression had been identified by the patient's NCD clinician. This underscores the importance of this study. Given that the study has shown that identifying depression in NCD clinic is a challenge, a short and valid screening tool like PHQ-9 can assist in the identification of patients with depression.

One of the strength of this study is that it is the first study to consider the validity of the PHQ-9 in non communicable disease clinics in Malawi, and the first to validate the PHQ-9 in Malawi. Another strength of the study, is the careful attention which was paid to the translation. The study also used a reference standard, the SCID for depression that had previously been translated and adapted for use in Chichewa. A limitation of this study is that the participants were drawn from only two specialized NCD clinics in Lilongwe which may not be representative of the wider population.

\section{Conclusion}

The validity, ease of administration and brevity of the PHQ-9 imply that it will be a valuable tool for identifying comorbid depression in patients with non communicable diseases using a cut-point of $\geq 9$. Clinicians in NCD clinics when choosing a tool for screening depression should consider the PHQ-9. The use of a validated PHQ-9 will be in line with the strategy of integrating depression management in chronic care clinics in Malawi. The findings support our planned use of the PHQ-9 as a screening tool in a pilot study to evaluate the effects of depression management on glycaemic control in non communicable diseases clinics in Malawi.

\section{Additional file}

Additional file 1: The Chichewa Patient Health Questionnaire (PHQ-9). (DOCX $18 \mathrm{~kb})$

\section{Abbreviations}

COMREC: College of Medicine Research and Ethics Committee; DSM: Diagnostic and Statistical Manual of Mental Disorders; NCDs: Noncommunicable Diseases; NPV: Negative Predictive Value; OCC: Overall Correct
Classification; PHQ-9: Patient Health Questionnaire-9; PPV: Positive Predictive Value; SCID: Structured Clinical Interview for DSM

\section{Acknowledgements}

The authors would like to express our sincere gratitude to DELTAS Africa Initiative through the African Mental Health Research Initiative (AMARI) for their financial support of this study. We would also like to thank the patients for their participation in the study. Finally, we would like to thank the research assistants who were responsible for consenting patients and data collection.

\section{Funding}

This study presents independent work supported through the DELTAS Africa Initiative [DEL-15-01]. The DELTAS Africa Initiative is an independent funding scheme of the African Academy of Sciences (AAS)'s Alliance for Accelerating Excellence in Science in Africa (AESA) and supported by the New Partnership for Africa's Development Planning and Coordinating Agency (NEPAD Agency) with funding from the Wellcome Trust [DEL-15-01] and the UK government. The views expressed in this publication are those of the author(s) and not necessarily those of AAS, NEPAD Agency, Wellcome Trust or the UK government.

\section{Availability of data and materials}

The datasets collected and/or analyzed during the current study are available from the corresponding author on reasonable request.

\section{Authors' contributions}

MU, ASM and BP were involved in the conceptualization of the study and supported the study implementation. MU analyzed the data and drafted the manuscript. BP supported the data analysis. RCS supported the study implementation. ASM, RCS and BP edited the manuscript. All authors read and approved the final manuscript.

\section{Ethics approval and consent to participate}

The study received ethical approval from the University of Malawi, College of Medicine Research and Ethics Committee (COMREC). All participants were provided with the necessary information. Written informed consent was given prior to data collection.

Consent for publication

Not applicable.

\section{Competing interests}

The authors declare that they have no competing interests.

\section{Publisher's Note}

Springer Nature remains neutral with regard to jurisdictional claims in published maps and institutional affiliations.

\section{Author details}

'Department of Mental Health, University of Malawi, College of Medicine, P/ Bag 360, Chichiri, Blantyre 3, Malawi. ${ }^{2}$ Department of Clinical Services, Ministry of Health, P. O. Box 30377, Capital City, Lilongwe 3, Malawi. ${ }^{3}$ Department of Public Health, University of Malawi, College of Medicine, P/ Bag 360, Chichiri, Blantyre 3, Malawi. ${ }^{4}$ Africa Center of Excellence in Public Health and Herbal Medicine, University of Malawi, College of Medicine, P/Bag 360, Chichiri, Blantyre 3, Malawi. ${ }^{5}$ Epidemiology Department, University of North Carolina at Chapel Hill Gillings School of Global Public Health, 135 Dauer Dr, Chapel Hill, NC 27599, USA.

Received: 18 September 2018 Accepted: 18 February 2019 Published online: 27 February 2019

\section{References}

1. Nakku JEM, et al. Validity and diagnostic accuracy of the Luganda version of the 9-item and 2-item patient health questionnaire for detecting major depressive disorder in rural Uganda. Global Mental Health. 2016;3.

2. Manea L, Gilbody S, McMillan D. Optimal cut-off score for diagnosing depression with the patient health questionnaire (PHQ-9): a meta-analysis. CMAJ : Canadian Medical Association Journal. 2012;184(3):E191-6. 
3. Mogga S, et al. Outcome of major depression in Ethiopia. Br J Psychiatry 2006;189(3):241.

4. Chisholm D, Saxena S. Cost effectiveness of strategies to combat neuropsychiatric conditions in sub-Saharan Africa and South East Asia: mathematical modelling study. BMJ. 2012;344.

5. Al-Otaibi $B$, et al. Depressive symptoms among Kuwaiti population attending primary healthcare setting: prevalence and influence of sociodemographic factors. Med Princ Pract. 2007;16(5):384-8.

6. Smarr KL, Keefer AL. Measures of depression and depressive symptoms: Beck Depression Inventory-II (BDI-II), Center for Epidemiologic Studies Depression Scale (CES-D), Geriatric Depression Scale (GDS), Hospital Anxiety and Depression Scale (HADS), and Patient Health Questionnaire-9 (PHQ-9). Arthritis care \& research. 2011;63(S11)

7. Mukeshimana M, Mchunu G. The co-morbidity of depression and other chronic non-communicable diseases: a review of literature on the epidemiology, diagnosis and health effects. Rwanda Journal. 2016;3(1):44-50.

8. Adewuya AO, Ola BA, Afolabi OO. Validity of the patient health questionnaire (PHQ-9) as a screening tool for depression amongst Nigerian university students. J Affect Disord. 2006;96(1):89-93.

9. Weobong B, et al. The comparative validity of screening scales for postnatal common mental disorder in Kintampo. Ghana. J Affect Disord. 2009;113.

10. Monahan PO, et al. Validity/reliability of PHQ-9 and PHQ-2 depression scales among adults living with HIV/AIDS in western Kenya. J Gen Intern Med. 2009;24.

11. Pence BW, et al. Validity of an interviewer-administered patient health Questionnaire-9 to screen for depression in HIV-infected patients in Cameroon. J Affect Disord. 2012;143(1-3):208-13.

12. Hanlon C, et al. Validity of brief screening questionnaires to detect depression in primary care in Ethiopia. J Affect Disord. 2015;186:32-9.

13. Bhana $A$, et al. The validity of the patient health questionnaire for screening depression in chronic care patients in primary health care in South Africa. BMC Psychiatry. 2015;15.

14. Stewart RC, et al. Validation of a Chichewa version of the self-reporting questionnaire $(\mathrm{SRQ})$ as a brief screening measure for maternal depressive disorder in Malawi, Africa. J Affect Disord. 2009:112(1-3):126-34.

15. Scholte WF, et al. Psychometric properties and longitudinal validation of the self-reporting questionnaire (SRQ-20) in a Rwandan community setting: a validation study. BMC Med Res Methodol. 2011;11(1):116.

16. Egede LE. Major depression in individuals with chronic medical disorders: prevalence, correlates and association with health resource utilization, lost productivity and functional disability. Gen Hosp Psychiatry. 2007;29(5):409-16.

17. Ziegelstein RC, et al. Patients with depression are less likely to follow recommendations to reduce cardiac risk during recovery from a myocardial infarction. Arch Intern Med. 2000;160(12):1818-23.

18. Moussavi $\mathrm{S}$, et al. Depression, chronic diseases, and decrements in health: results from the world health surveys. Lancet. 2007;370(9590):851-8.

19. Manjomo RC, et al. Managing and monitoring chronic non-communicable diseases in a primary health care clinic, Lilongwe, Malawi. Public Health Action. 2016;6(2):60-5.

20. Ciechanowski P, et al. Where is the patient? The association of psychosocial factors and missed primary care appointments in patients with diabetes. Gen Hosp Psychiatry. 2006;28(1):9-17.

21. Andreoulakis $\mathrm{E}$, et al. Depression in diabetes mellitus: a comprehensive review. Hippokratia. 2012:16(3):205-14.

22. Lin EHB, et al. Depression and advanced complications of diabetes. Diabetes Care. 2010;33(2):264.

23. Lloyd CE, et al. Prevalence and correlates of depressive disorders in people with type 2 diabetes: results from the international prevalence and treatment of diabetes and depression (INTERPRET-DD) study, a collaborative study carried out in 14 countries. Diabet Med. 2018.

24. Kroenke K, Spitzer RL, Williams JB. The PHQ-9: validity of a brief depression severity measure. J Gen Intern Med. 2001;16(9):606-13.

25. First MB, et al. Structured clinical interview for DSM-IV clinical version (SCIDI/CV. Washington DC: American Psychiatric Press; 1997.

26. Association, A.P., Diagnostic and statistical manual of mental disorders (DSM5๑). 2013: American Psychiatric Pub.

27. Association, A.P. Diagnostic and statistical manual of mental disorders (DSMIV). Washington, DC: American Psychiatry Association; 1994.

28. Buderer NMF. Statistical methodology: I. Incorporating the prevalence of disease into the sample size calculation for sensitivity and specificity. Acad Emerg Med. 1996;3(9):895-900.
29. Kauye F, Jenkins R, Rahman A. Training primary health care workers in mental health and its impact on diagnoses of common mental disorders in primary care of a developing country, Malawi: a cluster-randomized controlled trial. Psychol Med. 2014;44(03):657-66.

30. Ali G-C, Ryan G, De Silva MJ. Validated screening tools for common mental disorders in low and middle income countries: a systematic review. PLoS One. 2016;11(6):e0156939.

31. Lai P. Validating instruments of measure : is it really necessary? Malays Fam Physician. 2013;8(1):2-4.

32. Lowe $B$, et al. Comparative validity of three screening questionnaires for DSM-IV depressive disorders and physicians' diagnoses. J Affect Disord. 2004;78.

33. Mendenhall $\mathrm{E}$, et al. Depression and type 2 diabetes in low- and middleincome countries: a systematic review. Diabetes Res Clin Pract. 2014;103(2): 276-85.

34. Teshome HM, et al. The prevalence of depression among diabetic patients in Ethiopia: a systematic review and meta-analysis, 2018. Depress Res Treat. 2018;2018:8.
Ready to submit your research? Choose BMC and benefit from:

- fast, convenient online submission

- thorough peer review by experienced researchers in your field

- rapid publication on acceptance

- support for research data, including large and complex data types

- gold Open Access which fosters wider collaboration and increased citations

- maximum visibility for your research: over $100 \mathrm{M}$ website views per year

At $\mathrm{BMC}$, research is always in progress.

Learn more biomedcentral.com/submissions 